\title{
Editorial, COCOON 2007 Special Issue
}

\author{
Guohui Lin · Zhipeng Cai
}

Received: 28 February 2008 / Accepted: 9 June 2008 / Published online: 26 June 2008

(C) Springer Science+Business Media, LLC 2008

This special issue of the Algorithmica contains six selected papers that were presented at the Thirteenth Annual International Computing and Combinatorics Conference (COCOON 2007), held in Banff, Alberta, Canada, on July 16-19, 2007. COCOON 2007 received 154 final submissions for full consideration and 51 of them were accepted for podium presentations. Among these 51 accepted papers, 14 were invited to submit their full versions to two special issues. The substantial reviewing process accepted six of them to be included in this special issue. These six papers represent the best work in algorithms that were discussed in COCOON 2007, and we wish to disseminate them to the largest community.

We would like to thank the authors for submitting and revising their work, and PC members and additional referees for their careful reviewing.

Guest Editors

G. Lin $(\bowtie) \cdot$ Z. Cai

Department of Computing Science, University of Alberta, Edmonton, Alberta T6G 2E8, Canada e-mail: ghlin@cs.ualberta.ca

Z. Cai

e-mail: zhipeng@cs.ualberta.ca 